\title{
Gestão do Trabalho em Saúde
}

Wânia R. FERNANDES 1

\section{O TRABALHO EM SAÚDE}

Segundo Merhy e Franco1,2, o trabalho, como toda atividade humana, é um ato produtivo, que modifica e produz algo novo. Merhy1 desenvolveu o conceito de Trabalho vivo em ato, como sendo, o trabalho humano no exato momento em que é executado e que determina a produção do cuidado. Mas o trabalho vivo interage todo o tempo com instrumentos, normas, máquinas, formando assim um processo de trabalho, no qual interagem diversos tipos de tecnologias. Estas formas de interações configuram um certo sentido no modo de produzir o cuidado.

Vale ressaltar que todo trabalho é mediado por tecnologias. O arsenal tecnológico do trabalho em saúde é composto por: instrumentos (tecnologias duras); o saber técnico estruturado (tecnologias leve-duras) e as relações entre sujeitos que só têm materialidade em ato (tecnologias leves). Assim, pode haver a predominância da lógica instrumental como pode haver um processo em que os processos relacionais (interseçores) intervêm para um processo de trabalho com maiores graus de liberdade, tecnologicamente centrado nas tecnologias leves e leve-duras.

Não há trabalhador de saúde que consiga sozinho dar conta do complexo objeto do ato de cuidar: o mundo das necessidades de saúde. Deste modo, o trabalho de um técnico da saúde, de um profissional com formação universitária ou de um auxiliar, depende um do outro, depende desta troca, deste empréstimo.

A cena na qual é definido o modelo tecnológico de produção da saúde é permeada por sujeitos, com capacidade de operar pactuações entre si, de forma que a resultante dessas disputas é sempre produto da correlação de forças que se estabelece no processo. Essa pactuação, segundo MERHY1, não se dá apenas em processos de negociação, mas estrutura-se, muitas vezes, a partir de conflitos e tensões vividos no cenário de produção da saúde, seja na gestão ou na assistência.

O debate em torno do processo de trabalho tem se mostrado extremamente importante para a compreensão da organização da assistência à saúde e, fundamentalmente, de sua potência transformadora, particularmente quando nos debruçamos sobre a micropolítica de organização do trabalho. Verifica-se que, no modelo médico hegemônico, a distribuição do trabalho assistencial é dimensionada para concentrar o fluxo da assistência no profissional médico.

1 Doutora em Educação em Ciências e Saúde pela Universidade Federal do Rio de Janeiro. É pesquisadora do Núcleo de Estudos de Saúde Pública (NESP) da Universidade de Brasília. waniafer@hotmail.com 
No entanto, observa-se que o trabalho de todos os profissionais pode ser melhor aproveitado para cuidados diretos ao usuário, elevando assim a capacidade resolutiva dos serviços. Isso se faz, sobretudo, reestruturando os processos de trabalho e potencializando o "trabalho vivo em ato", como fonte de energia criativa e criadora de uma nova configuração na assistência à saúde.

Não há nenhum perfil de trabalhador que dê conta sozinho do mundo das necessidades de saúde, o objeto real do trabalho em saúde. Os trabalhadores universitários, técnicos e auxiliares são fundamentais para que o trabalho de um dê sentido ao trabalho do outro, na direção da verdadeira finalidade do trabalho em saúde: cuidar do usuário, o portador efetivo das necessidades de saúde.

\section{REESTRUTURAÇÃO PRODUTIVA EM SAÚDE}

A reestruturação produtiva é a resultante de mudanças no modo de produzir o cuidado, geradas a Gestão do Trabalho em Saúde Tempus Actas de Saúde Coletiva Políticas Públicas de Saúde: textos e contextos partir de inovações nos sistemas produtivos da saúde, que impactam o modo de fabricar os produtos da saúde e na sua forma de assistir e cuidar das pessoas e dos coletivos populacionais. Nem sempre novas formas de organizar o processo de trabalho resultam em modos radicalmente novos de produzir o cuidado que sejam capazes de impactar os processos de produção da saúde. As determinações para que uma reestruturação produtiva se realize são diversas. Os vários sujeitos, que estão ligados à área da saúde, disputam, nos lugares onde se decide sobre a organização da política e dos serviços de saúde, seus interesses distintos: corporativos, burocráticos, políticos e de mercado.

Como conseqüência dessas disputas, o modelo tecnológico de produção da saúde pode se caracterizar a partir de diversos dispositivos de mudança do modo de produzir saúde, sem no entanto mudar seu núcleo tecnológico, isto é, a mudança não é tão profunda no sentido de alterar a lógica da produção de saúde, a hegemonia centrada no trabalho morto (os mais comuns, hoje, como os centrados em procedimentos profissionais de cuidado, mais do que nas necessidades dos usuários) para outra centrada no trabalho vivo em ato, que se direciona pela centralidade do ato de cuidar do outro.

As mudanças, dos processos produtivos na saúde, podem ser verificadas se olharmos a partir da incorporação de novas tecnologias de cuidado, nos processos produtivos, nas outras maneiras de organização o processo de trabalho e, até mesmo, nas mudanças das atitudes dos profissionais, no modo de cuidar do outro.

Isto é, processos de subjetivação dos profissionais também podem determinar uma reestruturação produtiva, desde que impactem o modo de se produzir o cuidado. A reestruturação produtiva, como é processo, pode ocorrer de forma desigual e em diversos graus de mudança no interior dos processos de trabalho.

Merhy1 sugere outras categorias para designar e compreender as tecnologias de trabalho: // Tempus, actas de saúde colet, Brasília, 8(2), 115-123, jun, 2014. 
aquelascentradas em máquinas e instrumentos, chamadas de "tecnologias duras", as do conhecimento técnico, "tecnologias leve-duras", e as das relações, "tecnologias leves". Essas tecnologias operam o "trabalho morto" e o "trabalho vivo em ato", compondo assim os processos de produção da assistência à saúde, que determinam o núcleo tecnológico do trabalho.

Verifica-se que, para além das máquinas e do conhecimento técnico, há algo nuclear no trabalho em saúde, que são as relações entre os sujeitos e o agir cotidiano destes. Essa permanente atuação no cenário de produção da saúde configura, então, a "micropolítica do trabalho vivo em ato". Trata-se, sobretudo, do reconhecimento que o espaço onde se produz saúde é um lugar onde se realizam também os desejos e a intersubjetividade, que estruturam a ação dos sujeitos "trabalhador e usuário", individual e coletivo.

É possível haver, portanto, várias formas de reestruturação produtiva, sempre centradas na idéia de mudança nos processos de trabalho e no modo de produzir o cuidado. Mas se estas mudanças conseguem de fato alterar o núcleo tecnológico do cuidado, passando a operar centralmente as tecnologias leves, organizando um modo de produção centrado no trabalho vivo, com determinação dos sujeitos, trabalhador e usuário, que conduzem o processo de cuidado, isto pode configurar um modo de produção novo, ao qual conceituamos como transição tecnológica1,2.

Porém, este movimento não é só nesta direção do interesse do usuário, pois no próprio mercado da saúde, hoje, está instalada a disputa por uma transição tecnológica que aponta para outras formas de obtenção do lucro com o trabalho em saúde. Há uma disputa social importante entre o capital do complexo médico industrial, que se apóia em processos procedimento- centrados, e o capital financeiro das seguradoras e operadoras de planos de saúde, que obtém lucros quanto menos procedimentos realiza. Mas, nesse caso, o discurso em defesa da produção da saúde é instrumental, pois o objetivo central é o lucro com o cuidado de grupos populacionais que não fiquem doentes ou não consumam atos de saúde.

\section{POLÍTICAS E PROGRAMAS DO MS}

A Secretaria de Gestão do Trabalho e da Educação na Saúde (SGTES) é vinculada ao Ministério da Saúde e constituída pelo Decreto no 7.336, de 19 de outubro de 2010. Estruturada em dois departamentos, da Gestão da Educação na Saúde (DEGES) e da Gestão e da Regulação do Trabalho em Saúde (DEGERTS) e uma Diretoria de Programa, tem como objetivo "contribuir para a adequada formação, alocação, qualificação, valorização e democratização das relações de trabalho dos profissionais e trabalhadores de saúde".

Este objetivo vem ao encontro da superação do desafio nacional de ampliação da formação e da qualificação da força de trabalho em todos os setores econômicos, no qual a prestação de serviços essenciais, como os da saúde, se destaca como uma das prioridades na perspectiva de assegurar desenvolvimento e crescimento com justiça social e um país sem miséria. 
Todos os programas e ações desenvolvidos pela Secretaria de Gestão de Trabalho e da Educação na Saúde (SGTES), abaixo relacionados, convergem para a consolidação de políticas de valorização e de educação permanente do trabalhador da saúde.

\section{I - POLÍTICA NACIONAL DE EDUCAÇÃO PERMANENTE}

Marco Legal: Portaria GM/MS n 1.996/2007; Portaria GM/MS n 2.813/2008 ; Portaria GM/ $\mathrm{MS} \mathrm{n}^{\circ}$ 2953/2009; Portaria GM/MS n 4.033/2010; Portaria GM/MS nº 2200/2011.

Público Alvo: trabalhadores da rede de serviços do Sistema Único de Saúde (SUS).

Objeto: garantir a qualidade e resolubilidade da atenção à saúde prestada à população, por meio da implementação da educação permanente dos trabalhadores do SUS. A condução regional da Política é realizada pelas Comissões Intergestores Regionais - CIR com a participação das Comissões de Integração Ensino-Serviço (CIES), que são instâncias intersetoriais e interinstitucionais permanentes.

II - PROGRAMA DE PROFISSIONALIZAÇÃO DOS TRABALHADORES DE NÍVEL MÉDIO DA ÁREA DA SAÚDE - PROFAPS

Marco Legal: I - Saúde: Portaria GM/MS no 3.189/2009; Portaria GM/MS nº 1.626/2010; Portaria GM/MS n $1.307 / 2011$

II - Educação: Res. CEB Nº 04/1999 Público Alvo: trabalhadores de nível médio da Rede de Saúde do SUS.

I - Formação profissional técnica de nível médio/ Cursos Técnicos em: Análises Clínicas; Cipatologia; Enfermagem; Equipamentos Biomédicos; Hemoterapia; Órteses e próteses; Prótese Dentária; Radiologia; Saúde Bucal; e Vigilância em Saúde.

II - Qualificação Técnica (especializações, aperfeiçoamentos e atualizações) em áreas prioritárias, segundo demanda e necessidades locorregionais.

Objetivo: formar e qualificar trabalhadores de nível médio em áreas técnicas estratégicas para a saúde, visando à melhoria da atenção e da assistência à saúde do usuário do SUS.

III - PROGRAMA NACIONAL DE REORIENTAÇÃO DA FORMAÇÃO PROFISSIONAL EM SAÚDE - PRO- SAÚDE

Marco Legal: Portaria Interministerial no2.101/2005; Portaria no2.530/2005; Portaria Conjunta no 48/2006; Portaria Interministerial no3.019/2007; Portaria no07/2008; Portaria no1.282/2008; Portaria no 06/2012. 
Público Alvo: estudantes de graduação, docentes e profissionais dos serviços de saúde. Os projetos são desenvolvidos por Instituições de Educação Superior (IES) públicas ou privadas sem fins lucrativos, em parceria com Secretarias Municipais ou Estaduais de Saúde selecionadas pelos editais do Programa.

Objeto: profissionais com formação adequada para atender as necessidades de saúde da população brasileira.

Objetivo: o Pró-Saúde visa à reorientação da formação dos profissionais de saúde favorecendo mudanças curriculares segundo três eixos - orientação teórica, cenários de prática e orientação pedagógica com o objetivo de fortalecer a integração entre instituições de ensino e serviço público de saúde, com vistas à consolidação do Sistema Único de Saúde (SUS).

\section{IV - PROGRAMA NACIONAL DE TELESSAÚ- DE BRASIL REDES - TELESSAÚDE}

Marco legal: Portaria MS/GM nº 2.546/2011.

Público Alvo: profissionais e trabalhadores do SUS, na gestão e na assistência, nas práticas clínicas e processos de trabalho.

Objetivo: fortalecer a Atenção Básica.

Serviços Disponíveis: Teleconsultoria: consulta registrada e realizada entre trabalhadores, profissionais e gestores da área de saúde, com o fim de esclarecer dúvidas sobre procedimentos clínicos, ações de saúde e questões relativas ao processo de trabalho, podendo online e off-line.

Telediagnóstico: serviço autônomo para realizar serviços de apoio ao diagnóstico através de distância e temporal;

Segunda Opinião Formativa: resposta sistematizada, construída com base em revisão bibliográfica, nas melhores evidências científicas e clínicas originadas das teleconsultorias;

Tele-educação: conferências, aulas e cursos, ministrados por meio da utilização das tecnologias de informação e comunicação.

\section{V -PROGRAMA DE EDUCAÇÃO PELO TRABALHO PARA A SAÚDE - PET-SAÚDE}

Marco Legal: Portaria Interministerial $n^{\circ}$ 421/2010; Portaria Conjunta $n^{\circ}$ 2/2010; Portaria Conjunta $n^{\circ} 3 / 2010$; Portaria Conjunta n 6/2010; Portaria Interministerial $n^{\circ} 422 / 2010$; Portaria $n^{\circ}$ 4/2010; Portaria no 06/2012.

Público Alvo: profissionais de saúde do Sistema Único de Saúde (SUS), docentes e estudantes de graduação da área da saúde. Os projetos são desenvolvidos por Instituições de Educação Superior 
(IES) em parceria com Secretarias de Saúde.

Objeto: formação de grupos de aprendizagem tutorial para desenvolvimento de atividades em áreas estratégicas do Sistema Único de Saúde (SUS).

Objetivo: promover a integração ensino-serviço- comunidade, envolvendo docentes, estudantes de graduação e profissionais de saúde para desenvolvimento de atividades na rede pública de saúde, de forma que as necessidades dos serviços sejam fonte de produção de conhecimento e pesquisa em temas e áreas estratégicas do (SUS).

\section{VI - UNIVERSIDADE ABERTA DO SUS - UNA-SUS}

Marco Legal: Decreto Presidencial no 7.385/2010; Portaria Interministerial $n^{\circ} 1.277 / 2011$; Portaria Interministerial $\mathrm{n}^{\circ} 1.387 / 2011$

Público Alvo: profissionais da área da saúde do Sistema Único de Saúde (SUS).

Objeto: oferecer cursos e ações educacionais para atender às necessidades de capacitação e educação permanente dos trabalhadores do Sistema Único de Saúde - SUS, por meio do desenvolvimento da modalidade de educação à distância na área da saúde.

Objetivo: induzir e orientar a oferta de cursos de especialização, aperfeiçoamento e qualificação dirigidos aos trabalhadores do SUS; fomentar e apoiar a disseminação de meios e tecnologias de informação e comunicação; contribuir com a integração ensino- serviço na área de atenção à saúde e contribuir com a redução das desigualdades regionais, a partir da equalização da oferta de cursos.

\section{VII - PROGRAMA NACIONAL DE BOLSAS DE RESIDÊNCIA MULTIPROFISSIONAL E EM ÁREA PROFISSIONAL DA SAÚDE - RESIDÊNCIA}

Marco legal: A Portaria Conjunta MEC/MS nº 45/2007.

Público Alvo: hospitais universitários, hospitais de ensino, instituições de ensino superior e secretarias estaduais e municipais de saúde.

Objeto: financiamento de bolsas destinadas a profissionais de saúde interessados em se especializar na modalidade residência multiprofissional e em área profissional da saúde, em campos de atuação estratégicos para o Sistema Único de Saúde (SUS) e em regiões prioritárias do País, definidos em comum acordo com os gestores de saúde.

Objetivo: consolidar a Residência Multiprofissional e em Área Profissional da Saúde em campos de atuação estratégicos para o SUS.

VIII - PROGRAMA DE VALORIZAÇÃO DOS PROFISSIONAIS DA ATENÇÃO BÁSICA // Tempus, actas de saúde colet, Brasília, 8(2), 115-123, jun, 2014. 
PROVAB

Marco Legal: Portaria Interministerial nº 2.087/2011;

Público Alvo: médicos, enfermeiros e cirurgiões- dentistas.

Objeto: valorização, aperfeiçoamento e educação permanente do profissional que trabalha na $\mathrm{AB}$, como estratégia de aprimoramento das ações e dos serviços de saúde em áreas de difícil acesso e provimento ou de populações de maior vulnerabilidade.

Objetivo: capacitar o profissional de saúde e reduzir as desigualdades regionais existentes em nosso país no que diz respeito ao acesso à saúde. Além disso, o programa oferece aos profissionais participantes a oportunidade de conhecer diferentes realidades e de exercer a profissão onde a população mais necessita, fortalecendo a dimensão da relevância social de sua atuação.

\section{IX - PROGRAMA DE APOIO À FORMAÇÃO DE MÉDICOS ESPECIALISTAS EM ÁREAS ESTRATÉGICAS - PRO RESIDÊNCIA}

Marco Legal: A Portaria Conjunta $n^{\circ} 11$.

Público Alvo: hospitais universitários, hospitais de ensino, instituições de ensino superior e secretarias estaduais e municipais de saúde.

Objeto: Financiamento de bolsas destinadas a profissionais médicos selecionados em programas de residência, em diversas especialidades em regiões prioritárias para o SUS (Norte, Nordeste e Centro-Oeste).

Objetivo: apoiar a formação de médicos especialistas em regiões e especialidades prioritárias para o SUS.

\section{X - REDE OBSERVATÓRIO DE RECURSOS HUMANOS DE SAÚDE - OBSERVARH}

Marco Legal: Portaria MS/GM nº 14/2010.

Público Alvo: gestores do SUS, pesquisadores, docentes e estudantes da área da saúde que tenham interesse na área de Recursos Humanos de Saúde.

Objeto: estudos e pesquisas sobre Recursos Humanos de Saúde.

Objetivo: o propósito geral da Rede é produzir estudos e pesquisas, bem como propiciar o mais amplo acesso a informações e análises sobre a área de recursos humanos de saúde no país, facilitando melhor formulação, acompanhamento e avaliação de políticas e programas setoriais dessa área. Além disso, espera-se que a Rede também contribua para o desenvolvimento de 
$122 / /$

processos de controle social sobre a dinâmica e as tendências dos sistemas de educação e trabalho no campo da saúde.

\section{XI - PROGRAMADE QUALIFICAÇÃO E ESTRUTURAÇÃO DA GESTÃO DO TRABALHO E DA EDUCAÇÃO NO SUS - ProgeSUS}

Marco Legal: Portaria MD/GM nº 2.261/2006, que cria o ProgeSUS, está disponível no Portal do Ministério da Saúde.

Público Alvo: áreas de Gestão do Trabalho e da Educação das Secretarias de Saúde Estaduais,

Municipais e do Distrito Federal.

Objetivo: garantir maior articulação com as ações de democratização das relações do trabalho, estimulando as boas práticas de gestão e incentivando as ações inovadoras para a gestão do trabalho em nível local. Modernizar, aprimorar e criar áreas de gestão do trabalho e da educação nas Secretarias de Saúde.

\section{XII - DESPRECARIZAÇÃO DO TRABALHO EM SAÚDE - COMITÊ NACIONAL INTERINSTITUCIONAL DE DESPRECARIZAÇÃO DO TRABALHO NO SUS - DESPRECARIZASUS}

Marco legal: Portaria MS/GM n 2.430/2003.

Público alvo: trabalhadores em situação de trabalho precário.

Objeto: fórum constituído pelo MS, MPOG, Ministério do Trabalho, CONASS, CONASEMS e por representantes das entidades sindicais e empregadores do setor privado da saúde.

Objetivo: proposição de soluções para ampliação da proteção social aos trabalhadores na área desaúde e a reorganização dos trabalhadores do SUS em estruturas de carreiras para valorização profissional e melhorias das condições de trabalho no SUS.

\section{XIII - PORTAL SAÚDE BASEADA EM EVIDÊNCIAS}

Público Alvo: O site é voltado aos profissionais de saúde da rede pública das áreas de Biologia, Biomedicina, Educação Física, Enfermagem, Farmácia, Fisioterapia e Terapia Ocupacional, Fonoaudiologia, Medicina, Medicina Veterinária, Nutrição, Odontologia, Psicologia e Serviço Social.

Objeto: Utiliza a Epidemiologia Clínica; a Estatística; a Metodologia Científica; e a Informática para trabalhar a pesquisa; o conhecimento; e a atuação em Saúde. 
Objetivo: Promover a integração da experiência clínica às melhores evidências disponíveis, considerando a segurança nas intervenções e a ética na totalidade das ações. Pretende oferecer a melhor informação disponível para a tomada de decisão de intervenções preventivas, terapêuticas, diagnósticas e reabilitadoras.

\section{REFERÊNCIAS BIBLIOGRÁFICAS}

1- MERHY, E. E. Saúde: a cartografia do Trabalho Vivo. São Paulo: Hucitec, 2002.

2- FRANCO, T.B.; Processos de trabalho e transição tecnológica na saúde; Tese de Doutorado, Campinas (SP); Unicamp, 2003.

3- BRASIL. Ministério da Saúde. Secretaria de Gestão do Trabalho e da Educação na Saúde Secretaria de Gestão do Trabalho e da Educação na Saúde : SGETS : políticas e ações / Ministério da Saúde. Secretaria de Gestão do Trabalho e da Educação na Saúde. - Brasília : Ministério da Saúde, 2012.

Artigo apresentado em 21/01/14

Artigo aprovado em 22/03/14

Artigo publicado no sistema: 27/03/14 